\title{
Severe periodontal destruction caused by orthodontic elastic bands: A case report and review of the literature
}

\author{
Eudoxie Pepelassi ${ }^{1 *}$ and Dimitra E Tsarouchi ${ }^{2}$ \\ ${ }^{1}$ Associate Professor, Department of Periodontology, School of Dentistry, National and Kapodistrian University of Athens, Athens, Greece \\ ${ }^{2}$ Periodontist, Clinical Research Fellow at the Centre of Clinical Oral Research at the Institute of Dentistry, Queen Mary University of London, UK
}

\begin{abstract}
Background: The present article describes a case of extensive periodontal destruction caused by orthodontic elastic bands slipping apically into the periodontal ligament space.

Case description: Twelve years after placement of the orthodontic elastic bands the patient presented with severe iatrogenic damage to the periodontium of all four maxillary incisors rendering this, to the best of our knowledge, the longest period reported during which elastics were left lodged in situ. Removal of the bands was possible through the orifices of established sinuses and non-surgical periodontal treatment was attempted. At a subsequent visit, open flap debridement followed. At six months post-operatively, reduction of pockets, resolution of inflammation and sealing of the sinuses had occurred.
\end{abstract}

Conclusions: This report describes the pathology caused when orthodontic elastic bands get wedged into the periodontium and discusses the possible differential diagnosis. It emphasizes the importance of informing the patients on the correct use of elastics and suggests measures to prevent their displacement.

\section{Introduction}

Elastic bands have long been used in orthodontics in order to achieve a number of functions through intra- and intermaxillary traction [1]. The desired results include closure of diastemas, rotation of malpositioned teeth and correction of cross-bites [2-5]. In addition, elastics are frequently used in the form of orthodontic separators to create interdental space for the subsequent placement of metal bands $[6,7]$. Though they can prove valuable aids in achieving the above aims, problems can arise in the periodontal tissues when they are placed circumferentially around single/multiple teeth [1]. This inappropriate positioning in combination with a failure to secure them in place may lead to apical migration of the bands to the detriment of the periodontium. Similar complications were presented in the past, when patients with inadequate training were asked to apply and replace the elastics on their own as well as in cases where patients claimed to be unaware of the bands' presence $[3,4,6]$.

When searching the literature, cases describing the adverse effects from the slipping of elastic bands can be found as early as 1870 [813]. The overall clinical picture is that of gingival inflammation and loss of periodontal support around the affected teeth as a result of the band's gradual subgingival displacement. It has been recorded in both adults and children and can affect any tooth where elastics have been inappropriately placed, commonly maxillary incisors where attempts to close existing diastemas with the help of elastics have been reported [3].

\section{Case report}

A 25-year old, male patient presented to the Department of Periodontology, School of Dentistry, University of Athens, Greece, complaining of "sore gums' in the anterior maxilla and seeking treatment. His medical history was clear while his dental history revealed that he had started orthodontic treatment twelve years ago.
The orthodontist treated him for three years after which period the patient stopped attending his appointments and never completed the treatment.

The patient was subjected to thorough clinical and radiographic examination. In the anterior maxillary sextant, gingival inflammation localized around the central and lateral incisors with bleeding on probing and mild plaque accumulation were noticed. On the labial gingiva, distally to teeth $\# 8$ and $\# 10$, two sinus tracts leading to the roots' apical thirds and discharging serum on probing were detected (Figure 1). Probing of teeth $\# 7,8,9,10$ revealed severe clinical attachment loss, ranging from $9-12 \mathrm{~mm}$ and deep periodontal pockets at several sites (Figures 1 and 2). The radiographs confirmed the severe loss of alveolar bone around the aforementioned teeth but did not reveal a possible cause explaining it (Figure 3). Interestingly, there was no severe increase of mobility beyond grade I and no occlusal interferences detected. The periodontal tissues around the other teeth presented mild gingival inflammation but no other significant findings. While probing the sinus tracts the presence of two elastic bands, $15-20 \mathrm{~mm}$ long each, was detected and a diagnosis of iatrogenic periodontal destruction was made (Figure 4). Upon removal of the elastic bands through the established sinuses, the patient was subjected to non-surgical periodontal treatment, including oral hygiene instructions, cleaning of all teeth, scaling and root planing in the maxillary anterior sextant.

${ }^{\star}$ Correspondence to: Eudoxie Pepelassi, 2 Thivon street, Department of Periodontology, School of Dentistry, National and Kapodistrian University of Athens, 1527 Athens, Greece, Tel: (+30) 210-746-1223; E-mail: epepela@dent.uoa.gr

Key words: orthodontic treatment, elastic bands, elastics, periodontal destruction

Received: March 01, 2019; Accepted: March 25, 2019; Published: March 27, 2019 
Pepelassi E (2019) Severe periodontal destruction caused by orthodontic elastic bands: A case report and review of the literature

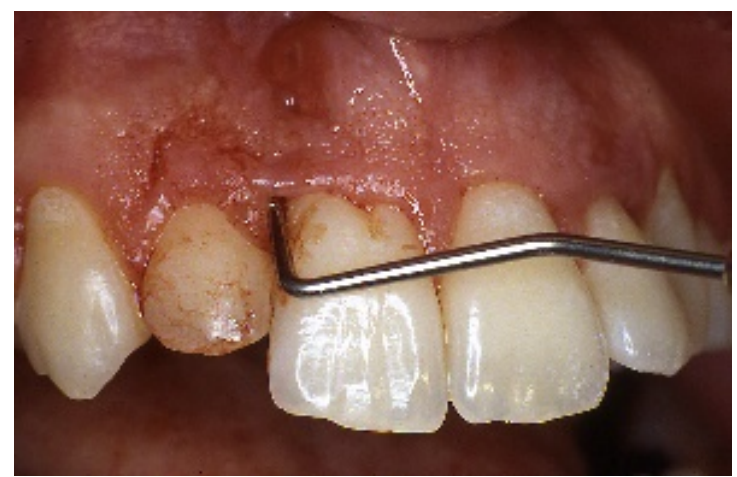

Figure 1. Clinical examination revealed severe clinical attachment loss, bleeding on probing and a buccal sinus present between teeth \#7 and \#8

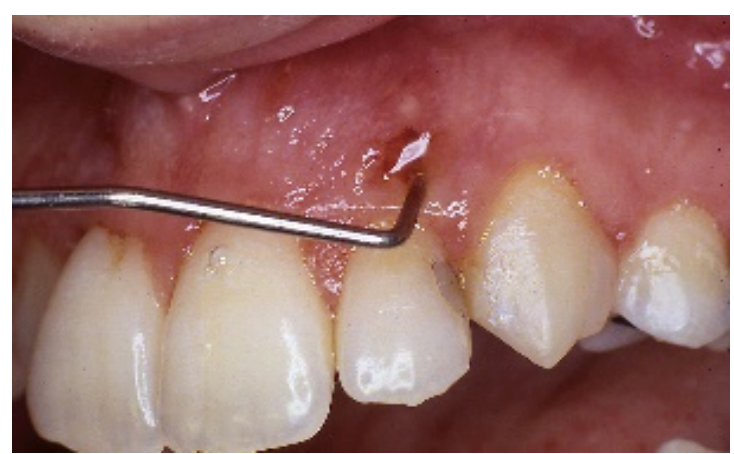

Figure 2. Clinical examination revealed severe clinical attachment loss, bleeding on probing and a sinus present buccally of tooth $\# 10$

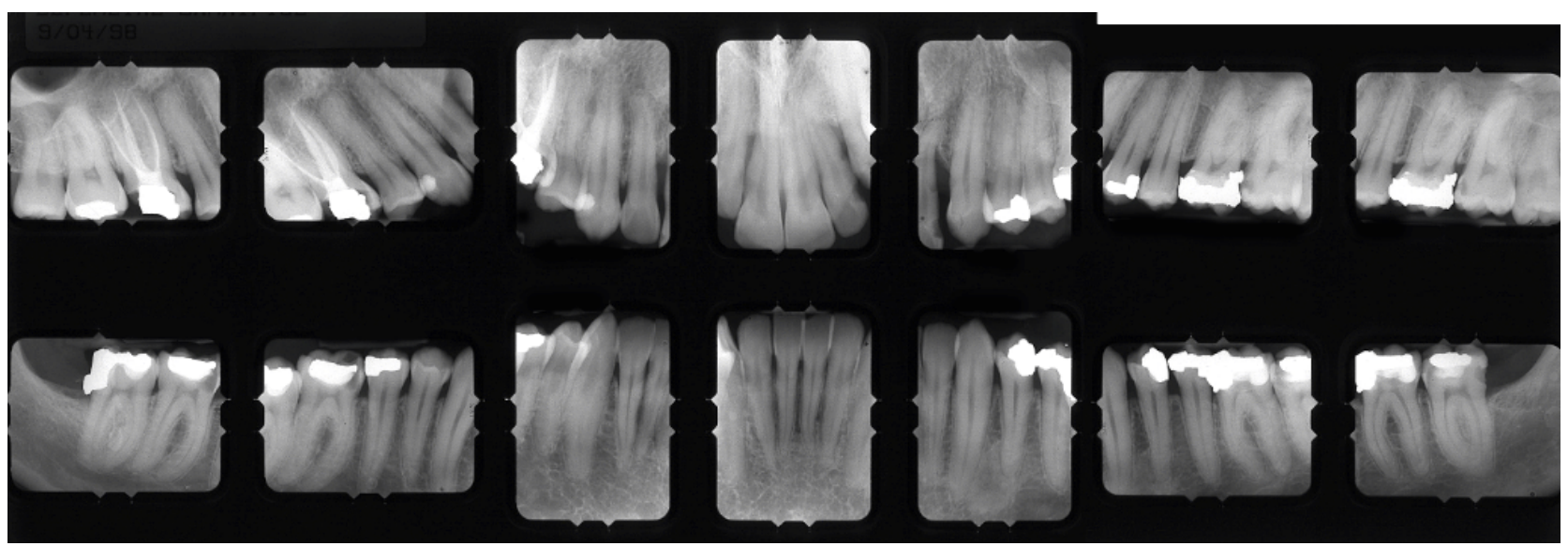

Figure 3. Radiographic examination revealed severe alveolar bone loss in the anterior sextant with evidence of angular defects between teeth \#7-8 and distally of \#11. There are normal alveolar bone levels at the remaining dentition

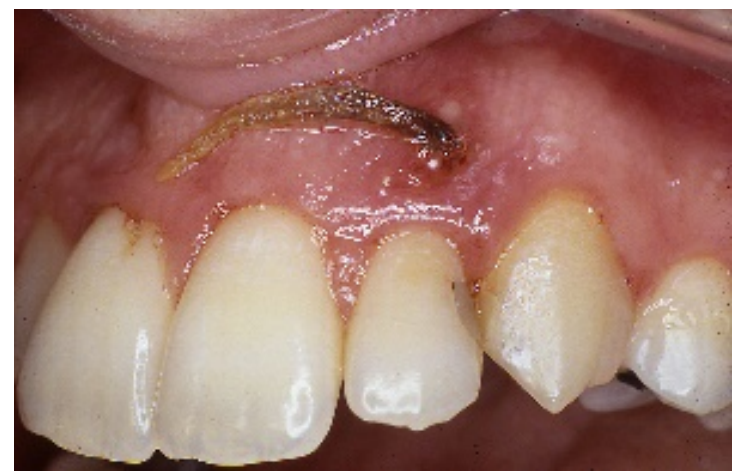

Figure 4. At the initial examination, a displaced orthodontic elastic band was detected and removed via the sinus opening with the help of a periodontal probe 
Re-evaluation at six weeks revealed slight reduction in pocket depths and BOP score as well as apical displacement of the gingival margin. Overall the clinical improvement was minimal with inflammation still persisting. As a result, a decision was made to explore the area surgically in order to check for foreign body remnants and perform open flap debridement of the defects. Labial and palatal intrasulcular incisions were performed starting at the distal aspect of tooth \#7 and extending distally of \#11 and full thickness mucoperiosteal flaps were raised. The area was inspected thoroughly, however no further signs of elastic bands were found. The granulation tissue was curetted away meticulously, the root surfaces were thoroughly planned, and the sinus tracts were excised. Upon closer inspection, severe alveolar bone loss had occurred around teeth $\# 7,8,9,10$ and 11 (Figure 5). There were angular osseous defects characterized by a combination of the number of osseous walls present that did not favor regenerative techniques (Figures 5 and 6). Despite the defects being extensive, reaching the apical third of teeth $\# 8,9,10$ every effort was made to preserve the affected teeth. Finally, the flaps were replaced and secured at their original position with interrupted 4.0 silk sutures (Figure 7).

On subsequent follow-up appointments during the course of the next six months, the area showed significant recovery with inflammation resolving, resulting to marked reduction of probing pocket depths. At six months, the sinus tracts had healed completely, and the values of the residual pockets ranged between 4 to $6 \mathrm{~mm}$. Further gingival shrinkage was noted as tissues contracted post-operatively (Figure 8). The patient was subsequently placed on regular recall and maintenance care.

\section{Discussion}

The adverse effects of elastic bands were already known by 1870 s and in the late $19^{\text {th }}$ and early $20^{\text {th }}$ century they were taken advantage of in order to induce exfoliation in haemophiliacs where minimal risk of bleeding was desired $[8,9]$. At the time it was believed that this alternative extraction method functioned based on the "inclined plane" principle: the elasticity of the band in combination with the conical root shape caused the rubber band to move towards the path of less resistance (i.e. smaller diameter) and thus move apically [9]. It was suggested that the above mechanical effect resulted in "anemic atrophy" of the periodontal tissues, whereby the pressure of the band constricts the vasculature and separates the tooth from the periodontium with minimal bleeding [8].

More recently, a series of experiments conducted in the 1970s (where rubber bands, silk and cotton floss ligatures were used to induce

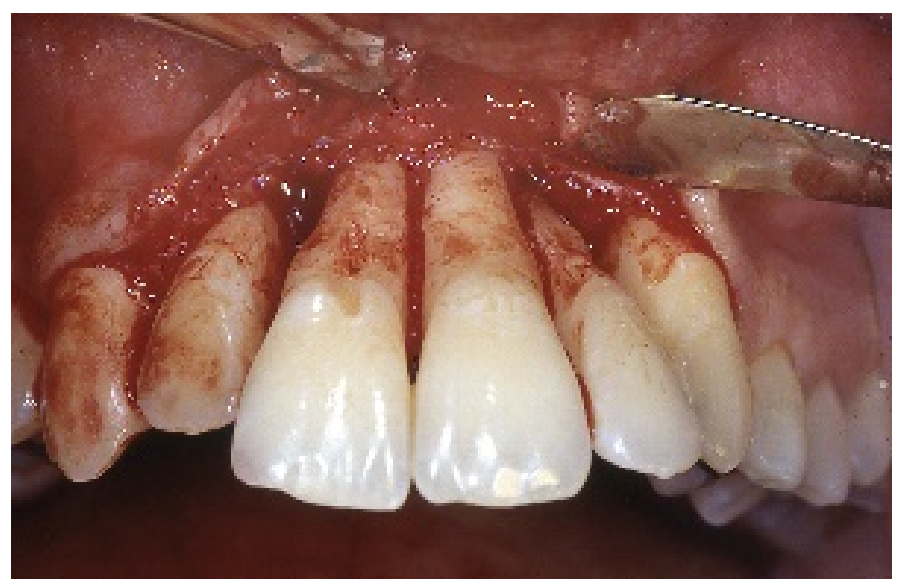

Figure 5. Labial aspect: open flap debridement revealed advanced loss of the alveolar bone supporting teeth \#7-11

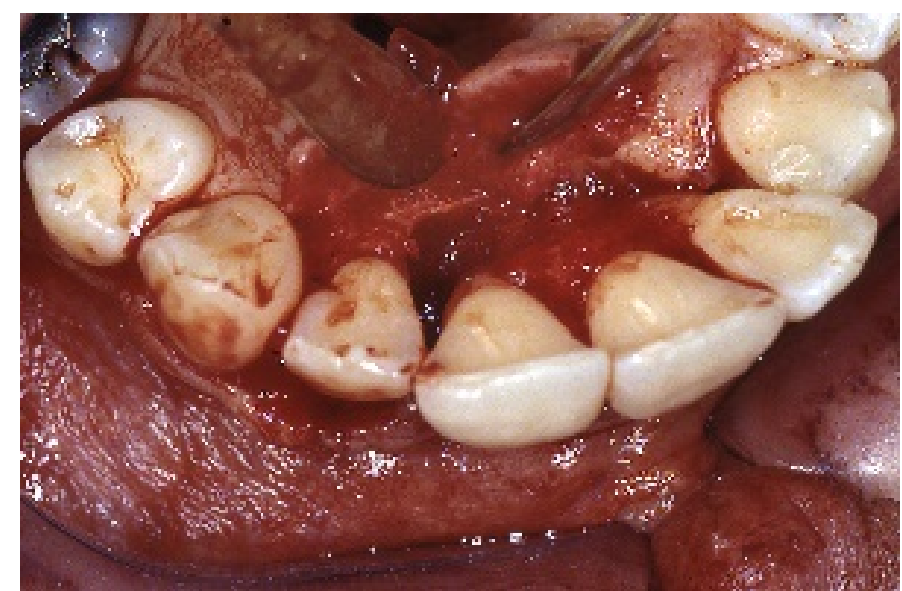

Figure 6. Palatal aspect: open flap debridement revealed advanced loss of the supporting alveolar bone, worsened at the areas mesial to tooth \#7, palatal to \#8 and distal to \#10

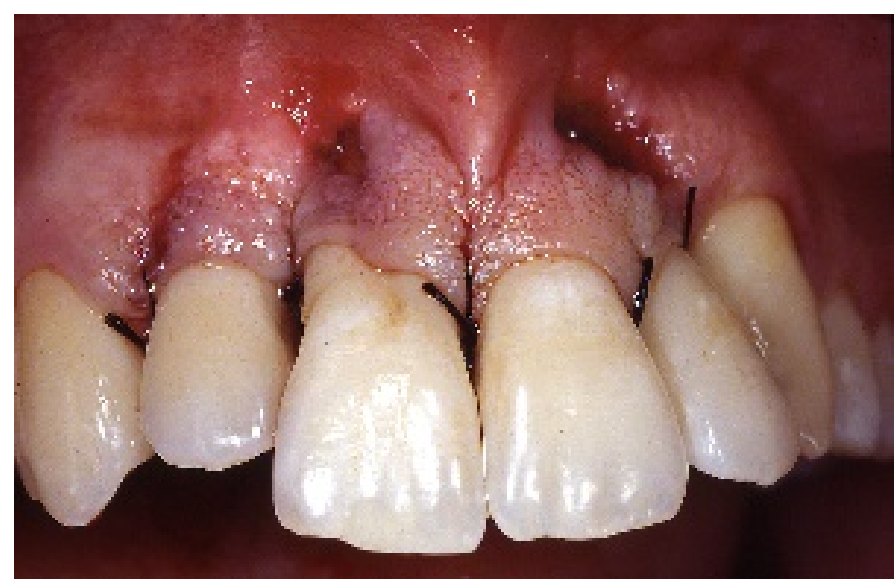

Figure 7. Following open flap debridement of the maxillary anterior sextant, the labial and palatal flaps were replaced at their original position using interrupted silk sutures

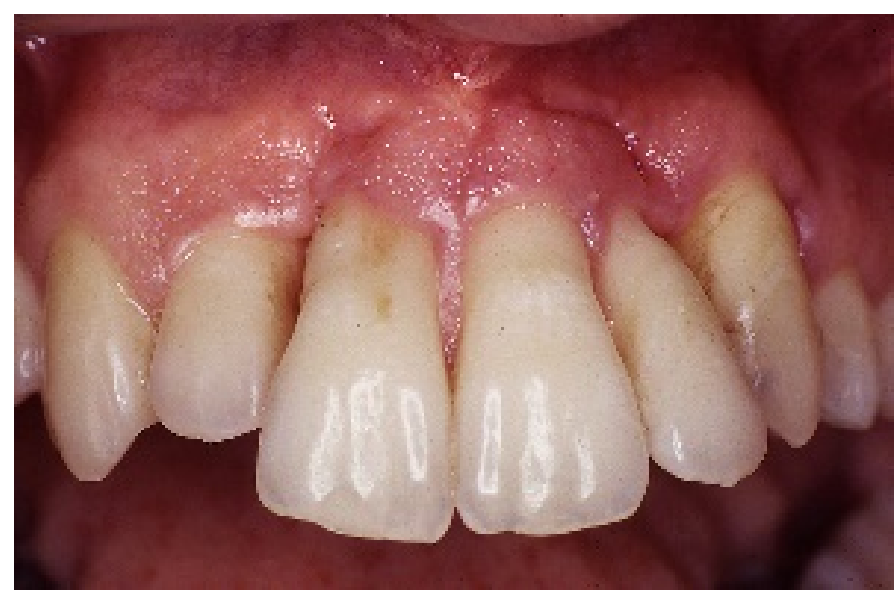

Figure 7. Following open flap debridement of the maxillary anterior sextant, the labial and palatal flaps were replaced at their original position using interrupted silk sutures

experimental periodontitis in animal studies) suggested that a likely cause for the periodontal destruction seen is the significant trapping of microbial plaque by these materials [14-18]. Microscopic examination allowed researchers to observe an increase in the subgingival population of gram-negative microorganisms and spirochetes as well as an influx 
of inflammatory cells in the tissues [16-19]. At the same time, traumainduced ulceration of the sulcular epithelium was observed co-existing with an acute inflammatory phase which in turn is postulated to induce and maintain osteoclastic activity leading to significant alveolar bone resorption $[14,18]$.

It is worth pointing out that the rapid progression of localized periodontal destruction, the absence of systemic disorders that could affect the periodontal status and the presence of an otherwise normal periodontium around the rest of the dentition may guide the clinician to consider alternative pathologies. Aggressive periodontitis, localized or generalized, is a plausible differential diagnosis especially since patients who present with findings of such iatrogenic periodontal destruction are frequently young individuals undergoing orthodontic treatment [10]. Consideration should be given to self-inflicted traumatic lesions, where further questioning should aim at revealing the causative habit [10]. Finally, in cases of extensive localized periodontal destruction one may be misled to consider the probability of some form of nonodontogenic tumour, such as eosinophilic granuloma, often affecting young individuals $[4,20]$. In such instances, sending off the curetted tissues for histopathological examination is an adjunct to the diagnosis.

Being able to detect the lodged foreign body based solely on periodontal probing and tactile sensation is undoubtedly clarifying matters but rather difficult to achieve, particularly in those instances where the band has migrated far into the apical third of the root. The most important points that can aid the clinician in suspecting the real cause of the problem are the existing history of orthodontic treatment and the rapid progression of signs and symptoms noted when the problem is detected at an early stage $[5,11]$. In the past radiographs were not particularly helpful since radiolucent elastics were used, however the introduction of radiopaque ones can greatly facilitate the diagnostic process.

\section{Conclusions}

The present case describes to the best of our knowledge the longest period for which elastic bands have remained undetected in the periodontium. It thus emphasizes the importance of ensuring patients are well informed of their presence, their function and correct method of placement as well as the potential consequences that can arise should bands suddenly go missing. It follows that good patient compliance with the instructions given is imperative whenever elastics are used. Additional preventive steps include documenting the bands' positions in the patient's file as well as the date of their removal. Periodontal examination performed routinely at orthodontic appointments helps detect such complications at an early stage and thus prevent them [6]. Furthermore, clinicians have denounced the solo use of elastic bands circumferentially around teeth $[1,12]$. Instead, when used as separators the elastics should be wedged in between teeth in order to create space $[6,7]$. Finally, when elastics are used to move teeth, several measures can be taken in order to secure them in place, such as anchoring them to brackets, metal hooks or orthodontic bands where available $[2,3,11]$.

\section{References}

1. Houston WJB, Stephens CD, Tulley WJ (1992) A textbook of orthodontics. 2nd ed Oxford Wright 288.

2. Vandersall DC (1971) Localized periodontitis induced by rubber elastic. J Am Dent Assoc 83: 1326-1328. [Crossref]

3. Vandersall DC, Varble DL (1978) The missing orthodontic elastic band, a periodonticorthodontic dilemma. J Am Dent Assoc 97: 661-663.

4. Zager NI, Barnett ML (1974) Severe bone loss in a child initiated by multiple orthodontic rubber bands: case report. J Periodontol 45: 701-704. [Crossref]

5. Kwapis BW, Knox JE (1972) Extrusion of teeth by elastics: report of two cases. J Am Dent Assoc 84: 629-631. [Crossref]

6. St George G, Donachie MA (2002) Case report: orthodontic separators as periodontal ligatures in periodontal bone loss. Eur J Prosthodont Restor Dent 10: 97-99.

7. Harrington Z, Darbar U (2007) Localised periodontitis associated with an ectopic orthodontic separator. Prim Dent Care 14: 5-6.

8. Dalitsch WW (1934) Dental extraction in hemophilia. JADA 21: 1804-1811.

9. Birch C, Snider FF (1939) Tooth extraction in hemophilia. JADA 26: 1933-1942.

10. Sanchez-Perez A, Moya-Villaescusa MJ, Gambín-Manzano MC, Bravo-Gonzalez LA (2006) Loss of a clinical attachment level in a child: differential diagnosis with aggressive periodontitis. Br Dent $J$ 201: 281-282.

11. Zilberman Y, Shteyer A, Azaz B (1976) Iatrogenic exfoliation of teeth by the incorrect use of orthodontic elastic bands. J Am Dent Assoc 93: 89-93. [Crossref]

12. Fui Lim K (1996) Latex elastic-induced periodontal damage: A case report on the subsequent orthodontic management. Quintessence International 27: 685-690.

13. Smidt A, Ehrlich J (1992) Iatrogenic exfoliation of a tooth following abuse of a rubber dam: a case report. Quintessence International 23: 15-18.

14. Schroeder HE, Lindhe J (1980) Conditions and pathological features of rapidly destructive, experimental periodontitis in dogs. J Periodontol 51: 6-19.

15. Lindhe J, Ericsson I (1978) Effect of ligature placement and dental plaque on periodontal tissue breakdown in the dog. J Periodontol 49: 343-350.

16. Kennedy JE, Polson AM (1973) Experimental marginal periodontitis in squirre monkeys. J Periodontol 44: 140-144.

17. Schroeder HE, Lindhe J (1975) Conversion of stable established gingivitis in the dog into destructive periodontitis. Arch Oral Biol. 20: 775-782.

18. Heijl L, Rifkin BR, Zander HA (1976) Conversion of chronic gingivitis to periodontitis in squirrel monkeys. J Periodontol 47: 710-716. [Crossref]

19. Caton JG, Zander HA (1975) Primate model for testing periodontal treatment procedures: I. Histologic investigation of localized periodontal pockets produced by orthodontic elastics. J Periodontol 46: 71-77.

20. Cawson RA, Odell EW (2002) Oral pathology and oral medicine. 7th ed. London: Churchill Livingstone 148 .

Copyright: (C2019 Pepelassi E. This is an open-access article distributed under the terms of the Creative Commons Attribution License, which permits unrestricted use, distribution, and reproduction in any medium, provided the original author and source are credited. 